\title{
Image Analysis of Soil Micromorphology: Feature Extraction, Segmentation, and Quality Inference
}

\author{
Petros Maragos \\ School of Electrical \& Computer Engineering, National Technical University of Athens, Athens 15773, Greece \\ Email:maragos@cs.ntua.gr
}

Anastasia Sofou

School of Electrical \& Computer Engineering, National Technical University of Athens, Athens 15773, Greece Email:sofou@cs.ntua.gr

\author{
Giorgos B. Stamou \\ School of Electrical \& Computer Engineering, National Technical University of Athens, Athens 15773, Greece \\ Email: gstam@softlab.ntua.gr \\ Vassilis Tzouvaras \\ School of Electrical \& Computer Engineering, National Technical University of Athens, Athens 15773, Greece \\ Email: tzouvaras@image.ntua.gr
}

\section{Efimia Papatheodorou}

Department of Biology, Ecology Division, Aristotle University of Thessaloniki, Thessaloniki 54006, Greece Email: papatheo@bio.auth.gr

\section{George P. Stamou \\ Department of Biology, Ecology Division, Aristotle University of Thessaloniki, Thessaloniki 54006, Greece Email: gpstamou@bio.auth.gr}

\section{Received 6 February 2003; Revised 15 December 2003}

\begin{abstract}
We present an automated system that we have developed for estimation of the bioecological quality of soils using various image analysis methodologies. Its goal is to analyze soilsection images, extract features related to their micromorphology, and relate the visual features to various degrees of soil fertility inferred from biochemical characteristics of the soil. The image methodologies used range from low-level image processing tasks, such as nonlinear enhancement, multiscale analysis, geometric feature detection, and size distributions, to object-oriented analysis, such as segmentation, region texture, and shape analysis.
\end{abstract}

Keywords and phrases: soilsection image analysis, geometric feature extraction, morphological segmentation, multiscale texture analysis, neurofuzzy quality inference.

\section{INTRODUCTION}

The goal of this research work is the automated estimation of the bioecological quality of soils using image processing and computer vision techniques. Estimating the soil quality with the traditional biochemical methods, and more specifically estimating those elements that are essential components for the soil fertility, is a difficult, time-consuming, and expensive process, which is, however, necessary for selecting and applying any management practice to land ecosys- tems. Our approach has been the development of an automated system that will recognize the characteristics relevant to the soil quality by computer processing of soilsection images and extraction of suitable visual features. Its final goals are double-fold: (1) quantification of the micromorphology of the soil via analysis of soilsection images and (2) correspondence of the extracted visual information with the classification of soil into various fertility degrees inferred from measurements performed biochemically on the soil samples. The overall system is shown in Figure 1. 


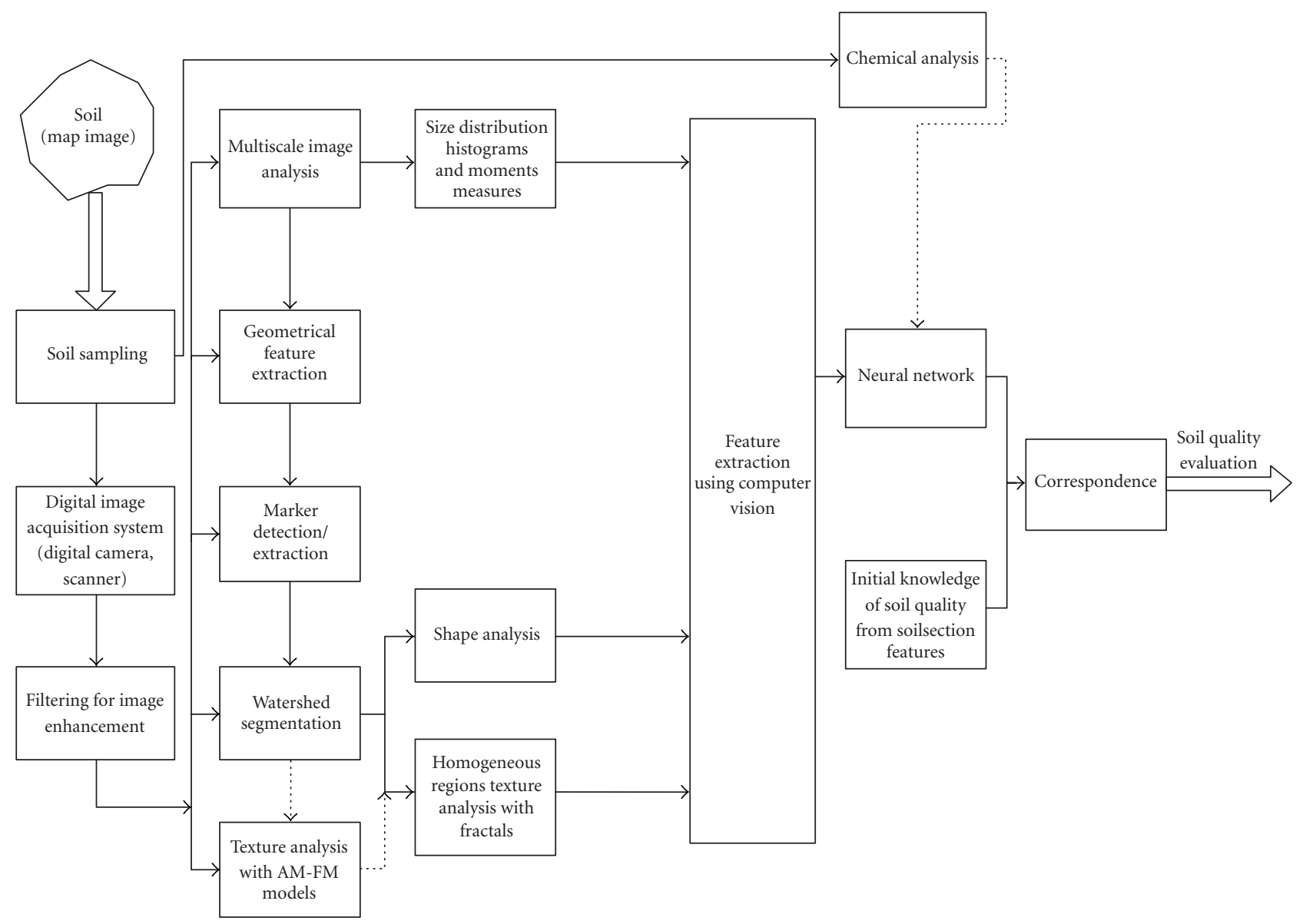

Figure 1: Overall system architecture.

In the image analysis part of this work, the above goals require solving a broad spectrum of problems in image processing and computer vision. Next, we list the most important of such problems (following a hierarchy from low-level vision to high-level vision) which we have investigated for detecting characteristics and extracting information from soilsection images: (1) enhancement of images; (2) feature detection; (3) multiscale image analysis; (4) statistical size distributions; (5) segmentation into homogeneous regions; (6) texture analysis; (7) shape analysis; and (8) correspondence of the features extracted from analyzing the soilsection images with the fertility grade of the soil inferred from its biochemical characteristics. The tools and methodologies that we have used for solving the above image analysis problems (1)-(7) include the following: (i) nonlinear geometric multiscale lattice-based image operators (of the morphological and fuzzy type) for multiscale image simplification and enhancement, extracting presegmentation features, size distributions, and region-based segmentation; (ii) nonlinear partial differential equations (PDEs) for isotropic modeling and implementing various multiscale evolution and visual detection tasks; (iii) fractals for quantifying texture and shape analysis from the viewpoint of geometrical complexity; (iv) modulation models for texture modeling from the viewpoint of instantaneous spatial frequency and amplitude components; and (v) topological and curvature-based methods for region shape analysis. Finally, methods of fuzzy logic and neural networks were investigated for the symbolic description and automated adaptation of the correspondence between the soilsection images and the bioecological quality of soil.

\section{SOIL DATA AND MICROMORPHOLOGY}

Soil data: the first phase of this work dealt with collecting soil samples both for performing biochemical measurements and for computer-based automated analysis of their images. During the phase of data collection, soil was sampled in mid September 2000 under the canopy of five characteristic shrubs of the Mediterranean (Greek) ecosystem (Juniperus sp., Quercus coccifera, Globularia sp., Erica sp. and Thymus $s p$.) as well as from the empty area among shrubs. Digital images of soilsections (of size in the order of about $20 \times 20 \mathrm{~mm}$ ) were formed using cameras and scanners at a resolution of 1200 dpi. Representative images from the six categories are 


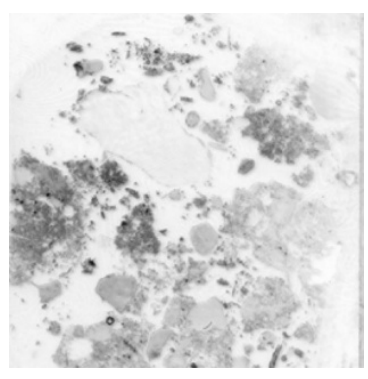

(a)

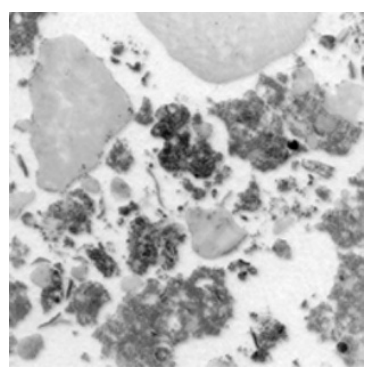

(d)

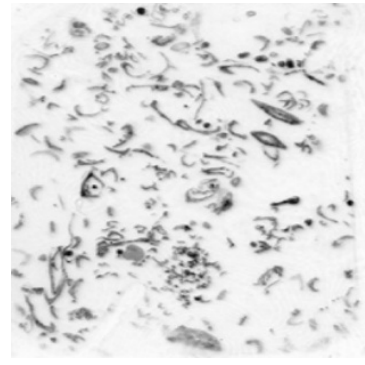

(b)

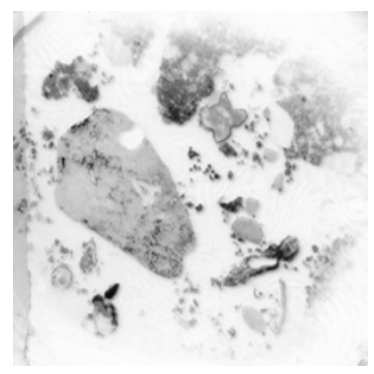

(e)

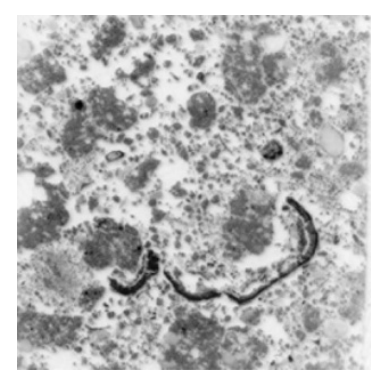

(c)

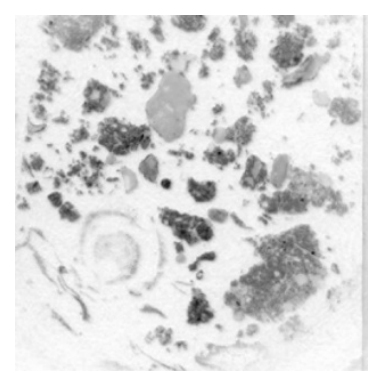

(f)

Figure 2: Characteristic soilsection categories. (a) Erica. (b) Thymus capitatus. (c) Juniperus oxycedrus. (d) Globularia alypum. (e) Quercus coccifera. (f) Void.

shown in Figure 2. The white regions correspond to air voids, while the dark regions to soil grains or aggregates.

Soil visual micromorphology: we summarize a few main concepts and definitions from [1]. The goal of soil micromorphology, as a branch of soil science, is the description, interpretation, and measurement of components, features, and fabrics in soils at a microscopic level. Basic soil components are the individual particles (e.g., quartz grains, clay minerals, and plant fragments) that can be resolved with the optical microscope together with the fine material that is unresolved into discrete individuals. Soil fabric deals with the total organization of a soil, expressed by the spatial arrangement of soil constituents, their shape, size, and frequency. Discrete fabric units are called pedofeatures. Soil structure is concerned with the size, shape, and spatial arrangement of primary particles and voids in both aggregated and nonaggregated material. Important characteristics of individual soil constituents, which are to be inferred by analyzing thin soilsections for describing soil fabric and structure, include: (1) size: classified into various scale bands, that is, micro $(1-100 \mu \mathrm{m})$, meso (100-1000 $\mu \mathrm{m})$, and macro (1-10 mm), (2) shape: 2D representation of 3D objects, (3) surface roughness/smoothness, (4) boundary shape, (an)isotropy, and complexity, (5) contrast: degree to which the feature being described is clearly differentiable from other features, and (6) sharpness: transition between the particular feature and other features. Many of these characteristics are a function of the orientation of components and the direction in which they are cut as well as of the magnification used.
Biochemical analysis: in parallel and independently from the analysis of soilsection images, biochemical measurements were also performed on the soil samples. Specifically, the soil samples were analysed for $\mathrm{C}$-microbial, $\mathrm{CO}_{2}$-evolution at $10^{\circ} \mathrm{C}$, fungal biomass by measuring ergosterol, bacterial substrate utilization (used as an index of bacterial activity) at $28^{\circ} \mathrm{C}$ for $120 \mathrm{~h}$, by using GN Biolog plates, rate of C-mineralization at $28^{\circ} \mathrm{C}$, C-organic, $\mathrm{N}$-organic, and $\mathrm{N}$ inorganic $\left(\mathrm{NH}_{4}\right.$ and $\left.\mathrm{NO}_{3}\right)$. These biochemical characteristics were used to infer the fertility grade of the soil.

\section{NONLINEAR \& GEOMETRIC IMAGE ANALYSIS}

\subsection{Enhancement and presegmentation feature detection}

The objective of image enhancement is to reduce the presence of noise, remove redundant information, and produce a smooth image that consists mostly of flat and large regions of interest. The methodology developed for the enhancement of soilsection images was based on the geometrical features and properties these images exhibit. Soilsection images have a great variety of geometrical features that can be either 1D such as edges or curves, or 2D such as light or dark blobs (small homogeneous regions of usually random shape) providing useful information for the evaluation of structure quality. Since shape, size, and contrast are features of primary importance, the image needs an object-oriented processing so that its structure is simplified but at the same time the remaining object-regions' boundaries are preserved. 
Three types of connected morphological operators ${ }^{1}$ that have such object-oriented properties are reconstruction and area openings and closings [3, 4] and levelings [5].

The (conditional) reconstruction opening $\rho^{-}(m \mid f)$ of an image $f$ given a marker signal $m \leq f$ can be obtained as follows:

$$
\rho^{-}(m \mid f)=\lim _{n \rightarrow \infty} \delta_{B}^{n}(m \mid f), \quad \delta_{B}(m \mid f)=(m \oplus B) \wedge f,
$$

where $\delta_{B}^{n}$ denotes the $n$-fold composition of the conditional dilation $\delta_{B}$ with itself and $B$ is a unit disk. The reconstruction closing is defined dually by iterating conditional erosions:

$$
\rho^{+}(m \mid f)=\lim _{n \rightarrow \infty} \varepsilon_{B}^{n}(m \mid f), \quad \varepsilon_{B}(m \mid f)=(m \ominus B) \vee f .
$$

The operations $\oplus$ and $\ominus$ denote the classic Minkowski dilation and erosion.

The area opening (closing) of a binary image at size scale $s \geq 0$ removes all the connected components of the image foreground (background) whose area is $<s$. Particularly, let the set $X=\bigsqcup_{i} C_{i}$ represent a binary image, where $C_{i}$ represent the connected components of $X$. The area opening output is $\alpha_{s}(X)=\bigsqcup_{j} C_{j}$ with area $\left(C_{j}\right) \geq s$, for all $j$. Any increasing binary operator can be extended to gray-level images via threshold superposition. Consider a gray-level image $f$ and its threshold binary images $f_{h}(x)$, where $h$ ranges over all gray levels. The value of $f_{h}(x)$ is 1 if $f(x) \geq h$ and 0 otherwise. Then, the gray-level area opening is defined as $\alpha_{s}(f)(x)=\sup \left\{h: \alpha_{s}\left(f_{h}\right)(x)=1\right\}$. If the image $f$ takes only nonnegative integer values $h \in\left\{0,1, \ldots, h_{\max }\right\}$, then

$$
\alpha_{s}(f)(x)=\sum_{h \geq 1} \alpha_{s}\left(f_{h}\right)(x) .
$$

Similarly, we can define the area closing of $f$ by duality as $\beta_{s}(f)=h_{\max }-\alpha_{s}\left(h_{\max }-f\right)$.

The levelings are a powerful class of self-dual connected operators [5]. The leveling $\Lambda(m \mid f)$ of a reference image $f$ given a marker $m$ can be obtained either from (i) a specific composition $\rho^{+}\left(\rho^{-}(m \mid f) \mid f\right)$ of a reconstruction opening followed by a reconstruction closing, where the former result is used as the marker of the latter or (ii) as the limit (as $t \rightarrow \infty$ ) of a scale-space function $u(x, t)$ generated by the following PDE [5]:

$$
\frac{\partial u}{\partial t}=-\operatorname{sign}[u(x, t)-f(x)]\|\nabla u\|
$$

with initial condition $u(x, 0)=m(x)$.

Based on the demands of the specific application, we have found that the following two systems of morphological con-

\footnotetext{
${ }^{1}$ Whenever we refer to morphological operators we will mean them in the lattice-theoretic sense [2]. Namely, consider the complete lattice $\mathcal{L}$ of real-valued image signals equipped with the partial ordering $f \leq g$, the supremum $\vee$, and the infimum $\Lambda$. Then, dilation (erosion) is any operator that distributes over $\bigvee(\Lambda)$. Further, opening (closing) is any operator that is antiextensive (extensive), increasing, and idempotent.
}

nected filters were the most suitable family of operators for enhancement and simplification of the soilsection images: (1) alternating sequential filters (ASFs), consisting of multiscale alternating openings and closings of the area type or reconstruction type; (2) multiscale levelings [5]. Scale in both cases was obtained by varying the scale of the marker signal.

Furthermore, we have developed generalized morphological operators by using concepts from lattice morphology and fuzzy sets. Specifically, we defined as lattice-fuzzy dilation

$$
\delta_{\mathrm{fuz}}(f)(x)=\bigvee_{y} T[f(y), g(x-y)]
$$

formed as supremum of a fuzzy intersection norm $T$, which can be the minimum, product or any other parametric triangular norm (T-norm) [6]. Replacing the sup with infimum and $T$ with its adjoint fuzzy implication operation yields a lattice-fuzzy erosion $\varepsilon_{\mathrm{fuz}}$ such that the pair $\left(\varepsilon_{\mathrm{fuz}}, \delta_{\mathrm{fuz}}\right)$ forms a lattice adjunction [2]. This guarantees that their composition will be a valid algebraic opening or closing. The power, but also the difficulty, in applying these fuzzy operators to image analysis is the large variety of fuzzy norms and the absence of systematic ways in selecting them. Towards this goal, we have performed extensive experiments in applying these fuzzy operators to various nonlinear filtering and soil image analysis tasks, attempting first to understand the effect that the type of fuzzy norm and the shape and size of structuring function have on the resulting new image operators. In general, we have observed that the fuzzy operators are more adaptive and track closer the image peaks/valleys than the corresponding flat morphological operators of the same scale. Details can be found in [7].

After the enhancement follows the feature extraction stage, such as estimation of an edge gradient which can provide information about critical zones and regions of interest that are present in the soilsection image $f$. A simple and efficient scheme is the morphological gradient $f \oplus B-f \ominus B$. Further, we have developed some new fuzzy gradients of the type $\min \left[\delta_{\mathrm{fuz}}(f), 1-\varepsilon_{\mathrm{fuz}}(f)\right]$ which yielded sharper image edges [7].

Using the aforementioned edge gradients and other nonlinear object-oriented operators we extract 2D features such as dark or light blobs that indicate the presence of objectsregions. Such operators are the generalized top-hat transform defined as the residual $\psi^{+}(f)=f-\alpha(f)$, as well as its dual bottom-hat transform $\psi^{-}(f)=\beta(f)-f$. The operators $\alpha$ and $\beta$ are generalized openings and closings, respectively, of the Minkowski, area, or reconstruction type.

\subsection{Granulometric size distributions}

Using Matheron's theory of sizing and granulometries, the classic Minkowski openings and closings by multiscale convex sets can yield size distributions of images [8]. The corresponding size histograms (a.k.a. "pattern spectra") have been very useful for shape-size description of images and for detecting critical scales $[8,9]$. The size histograms are especially important for analyzing soilsection images where multiscale size and shape of the soil components play a central role. For 
this application, we have developed generalized granulometric size distributions by using multiscale openings and closings of the area and reconstruction type [10].

Let $\alpha_{s}$ and $\beta_{s}$ denote families of multiscale openings and closings, respectively, which depend on a scale parameter $s \geq$ 0 and vary monotonically as the scale varies:

$$
s<r \Longrightarrow \alpha_{s}(f) \geq \alpha_{r}(f), \quad \beta_{s}(f) \leq \beta_{r}(f) .
$$

By measuring the volume $\operatorname{Vol}(\cdot)$ under the surface of these multiscale filterings of $f$, we can create the granulometry

$$
G_{f}(s)= \begin{cases}\operatorname{Vol}\left(\alpha_{s}(f)\right), & s \geq 0 \\ \operatorname{Vol}\left(\beta_{-s}(f)\right), & s<0\end{cases}
$$

Due to (6), the granulometry $G_{f}(s)$ decreases as $s$ increases. Further, after some appropriate normalization [9], it can become the size distribution of a random variable whose value is related to the size content of $f$. The derivative of this distribution yields a size density which behaves like the probability density function of this random variable. Ignoring this size density, for notational simplicity, the normalizing factor yields a nonnegative function $P_{f}(s)=-d G_{f}(s) / d s$. This unnormalized size density is also called "pattern spectrum" due to its ability to quantify the shape-size content of images [9]. For discrete images $f$, we use integer scales $s$, the granulometry $G_{f}(s)$ is obtained as above by defining $\operatorname{Vol}(f)$ as the sum of values of $f$, and the size density $P_{f}(s)$ is obtained by using differences instead of derivatives:

$$
P_{f}(s)=G_{f}(s)-G_{f}(s+1) .
$$

In the discrete case, we call $P_{f}(s)$ a size histogram. Now, we have examined three types of size histograms for soilsection images by using three corresponding types of multiscale openings and closings: (1) classic Minkowski openings $\alpha_{s}(f)=(f \ominus s B) \oplus s B$ and closings $\beta_{s}(f)=(f \oplus s B) \ominus s B$ by flat disks of radii $s$; $(2)$ reconstruction openings $\rho^{-}(f \ominus s B \mid f)$ and reconstruction closings $\rho^{+}(f \oplus s B \mid f)$ with multiscale markers; and (3) area openings and closings where the varying scale $s$ coincides with the area threshold below which components are removed by the filter.

All the above multiscale openings and closings obey the threshold superposition. The pattern spectrum inherits this property [9]. Thus, if a discrete image $f$ assumes integer values $h \in\left\{0,1, \ldots, h_{\max }\right\}$, then

$$
P_{f}(s)=\sum_{h \geq 1}^{m} P_{f_{h}}(s),
$$

where $f_{h}$ is the threshold binary image obtained from $f$ by thresholding it at level $h$. The above property allowed us to develop in [10] a fast algorithm for measuring the generalized size histograms, because the size histograms based on reconstruction and area openings become extremely fast when applied to binary images since we essentially need just to label the connected components of the binary image and count their areas. Then the total size histogram results as the sum of the histograms of all the threshold binary images.
The aforementioned granulometric analysis based on classic and generalized openings is applied to the characterization and description of the size content of soilsection images. Typical experimental results are shown in Figure 3, where the closings yield the size distribution of the dark image objects, that is, the soil grains or aggregates. In general, the classic size histogram based on Minkowski granulometries informs us on how the (volume) combination of size and contrast is distributed among soil components across many scales. Isolated spikes indicate the existence of objects with components at those scales. As Figure $3 \mathrm{c}$ shows, the size histogram based on reconstruction closings offers a better localization of the object sizes since the histogram presents abrupt peaks at the scales where large connected objects exist. The area closing size histogram of a binary image contains spikes only at scales equal to areas of binary components existing in the image. The area size histogram of a graylevel image, as in Figure 3d, is a superposition of the area histograms of its threshold binary images, as property (9) predicts.

\subsection{Texture analysis}

Objects or regions of interest in soilsection images often exhibit a considerable degree of geometrical complexity in their boundary or surface. Such sets can be modeled as fractals. The degree of surface roughness, measured via its fractal dimension, can serve as a useful descriptor for texture analysis. In our work, we estimate the fractal dimension $D$ of homogeneous regions using multiscale surface covers computed via multiscale flat morphological erosions and dilations. Specifically, $D=\lim _{r \downarrow 0} \log \operatorname{Vol}[f \oplus r B-f \ominus r B] / \log (1 / r)$. The estimated fractal dimension can be used as a measure of local texture roughness of soilsection images and can help with their classification.

We have also studied the texture of soilsection images using 2D AM-FM models and energy demodulation algorithms [11]. A texture image is locally modeled as a 2D AM-FM signal $a(x, y) \cos [\phi(x, y)]$, meaning that it can be parametrized by a local spatial frequency vector $\left(\omega_{x}, \omega_{y}\right)=(\partial \phi / \partial x, \partial \phi / \partial y)$ and a local intensity amplitude (contrast) $|a(x, y)|$. These $2 \mathrm{D}$ instantaneous spatial amplitude and frequency signals are the components of the 2D AM-FM image model. Based on the fact that local spatial frequencies have higher absolute values where greater alterations in texture occur, we can distinguish the different texture regions that are present in soilsection images. Using a $2 \mathrm{D}$ energy-based demodulation algorithm with relatively low computational complexity, based on a $2 \mathrm{D}$ energy-tracking operator $\Psi(f)=\|\nabla f\|^{2}-f \nabla^{2} f$, we were able to estimate the constituent signals $|a|, \omega_{x}, \omega_{y}$ of the model and presegment the soilsection image in distinct texture areas.

\section{SEGMENTATION}

Segmentation of soilsection images is a very important task for automating the measurement of the grains' properties as well as for detecting and recognizing objects in the soil, important for its bioecological quality. It proves to be difficult to achieve due to the low contrast, complex structure, and 


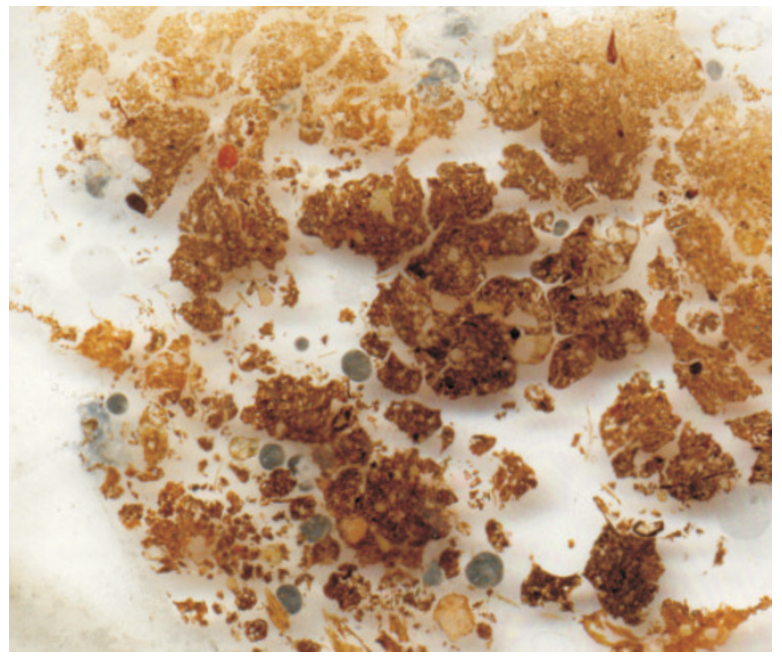

(a)

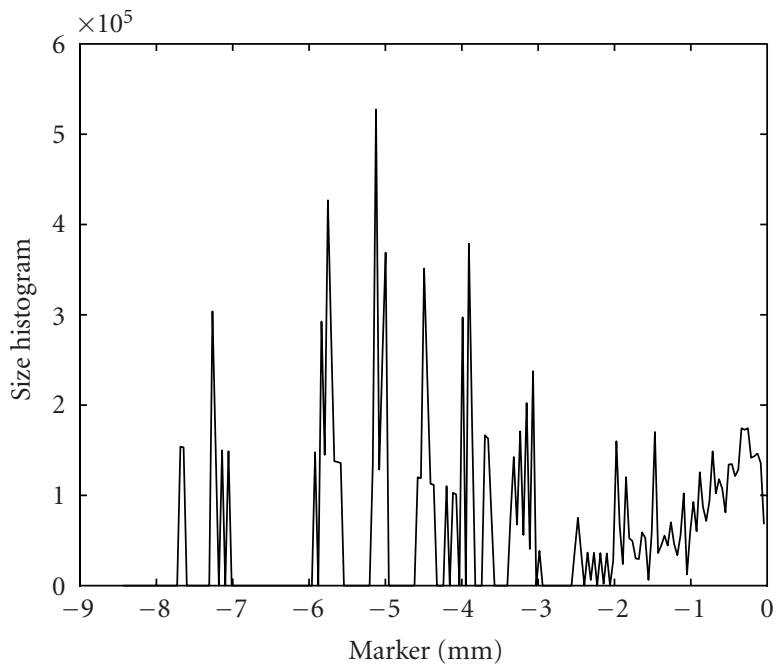

(c)

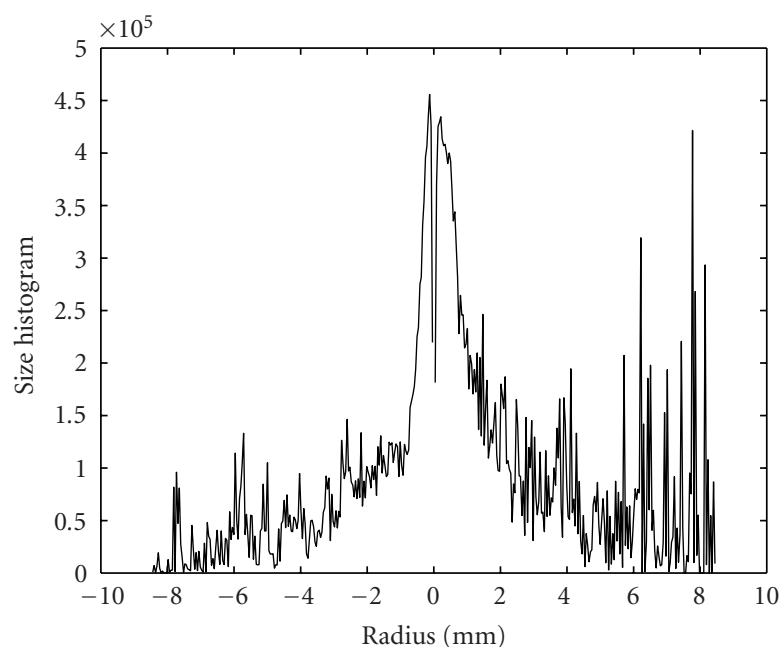

(b)

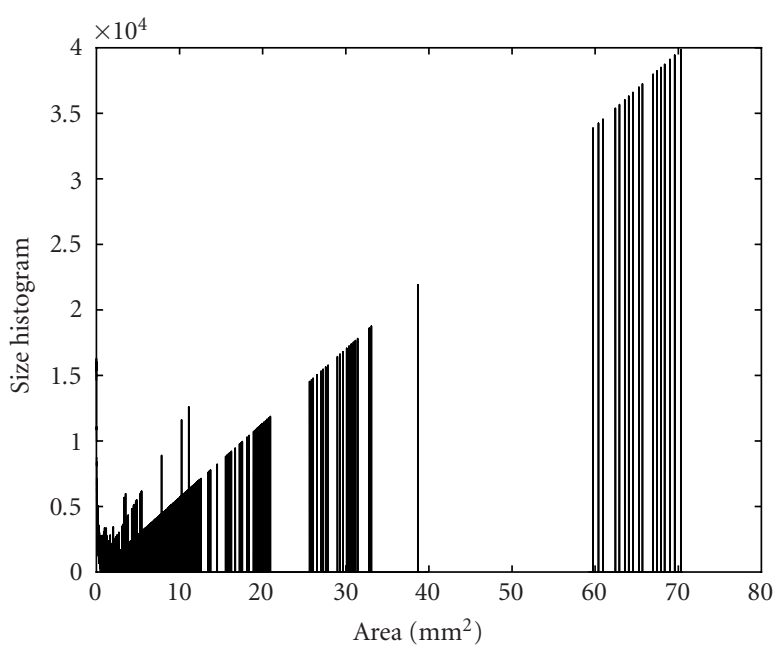

(d)

Figure 3: Size histograms for a soilsection image. (a) Original image $(405 \times 479$ pixels, $20.3 \times 17.2 \mathrm{~mm})$. (b) Size histogram based on flat Minkowski openings/closings. (c) Size histogram based on reconstruction closings. (d) Size histogram based on area closings.

often overlapping components present in these images. A well-known segmentation methodology in the field of mathematical morphology is the watershed approach [12], which is the preferred solution for the segmentation of soilsection images. The segmentation task can be divided into three different stages: (a) preprocessing and image enhancement, (b) region-feature extraction, and (c) watershed transform [13].

As described in Section 3.1, stage (a) is of critical importance since its output strongly influences the segmentation results. Its objective is to reduce the presence of noise and make the image easier to segment by removing useless information, thus producing an image that consists mostly of flat and large regions. Since we are interested in object boundaries, the images need to be processed in such a way that their structure is simplified, the objects' interior texture is smoothed while the relevant contour information is accurately preserved. Preservation of object boundaries is the main property of connected operators, described in Section 3.1, which differentiates them from other operators that perform their function locally, thus affecting region boundaries. Connected operators do not remove some frequency components (like linear filters do) or some small-size structures (like median filters or simple openings and closings do), but what they actually do is removing and merging flat zones. The preprocessing was based on reconstruction filters (1), (2) and area filters (3). Reconstruction openings remove entire bright components that are not marked by the markers, filling up the voids in soil grains or clusters and 


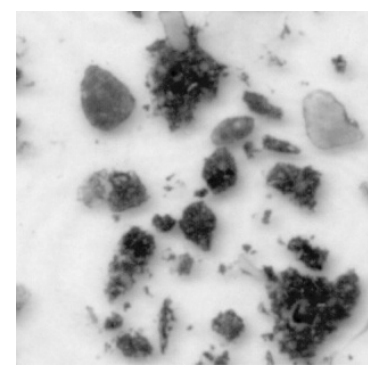

(a)

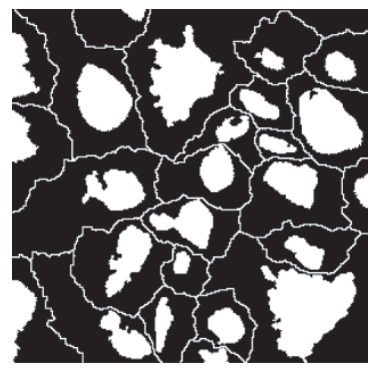

(c)

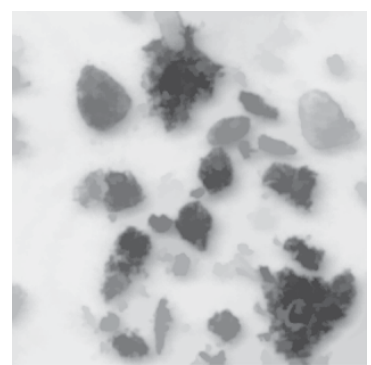

(b)

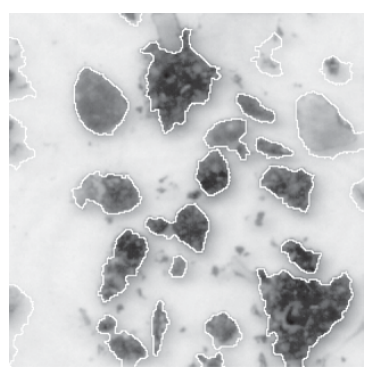

(d)
Figure 4: Segmentation stages: (a) original image, (b) enhanced image, (c) markers, and (d) segmented image.

making them more flat and uniform. Similarly, reconstruction closings remove dark components that are disjoint from the markers, eliminating very small soil grains and dark regions, making the background more uniform. The image can be further simplified by applying area openings and closings. An area closing with relatively low area threshold suppresses small dark regions, whereas an area opening with relatively high threshold merges flat regions inside the boundaries of soil grains, making the grains look darker and more uniform. In this way, arbitrarily shaped image components with area smaller than a given threshold are suppressed and the resulting image consists mostly of flat regions. The outcome of this stage can be viewed in Figure 4b.

At stage (b), the goal is to extract some special features from the simplified image such as small region seeds, called markers, which will be used as the starting points for the flooding process. The markers should be indicative of the regions where the objects of interest exist. Using the edge gradients mentioned in Section 3.1 and performing nonlinear object-oriented processing on the image, we extract region features such as contrast grain markers via the following procedure. First, we perform a reconstruction closing (2) to the simplified image $f$ (obtained after the enhancement stage) by using as marker $m=f+h$, the simplified image incremented by a constant $h$. The simplified image $f$ is subtracted from the reconstructed image $\rho^{+}(f+h \mid f)$, and the resulting image residue is thresholded at a level about $h / 2$. The obtained binary image is the set of markers that are included in the clusters of soil grains. These inside markers specify the location of the soil grains of a certain contrast that produce val- leys of contrast depth $h$. The size and shape of region markers are not critical for the segmentation, but only their location and existence. These features are of extreme importance since they specify the location of soil grains and clusters of a certain contrast and are used as segmentation seeds. In order to segment the image successfully, another set of markers is needed. This set is called outside markers and corresponds to the background of the image. The marker for the background is extracted by flooding the filtered soilsection image using as sources the inside markers. The resulted watershed line is the outside connected marker (background marker). The final set of markers is the union of the two sets detected previously, markers $=$ inside markers $\cup$ outside markers, presented in Figure 4c.

At stage (c), the watershed transform is applied on the morphological gradient of the enhanced image. It can be viewed as the process of flooding a topographic surface using the markers as sources. The watershed construction grows the markers until the exact contours of the objects are found. The watershed transformation is implemented via hierarchical queues using an ordering relation for flooding [12]. Figure 4 shows an example of our results from segmenting a soilsection image using the above methodologies. As shown in Figure 4d, most of the soil grains are detected. The ones that are missed are of small size and low contrast compared to their local background. This was expected due to the specific filtering that was performed on the image.

\section{POSTSEGMENTATION VISUAL FEATURE EXTRACTION}

After the segmentation is completed, the obtained regions are further processed in order to determine some postsegmentation features related to size, shape, and texture. Initially, we measure the area of global soil structure in comparison to void. In addition, various other local region descriptors are computed such as the area, perimeter, equivalent diameter, eccentricity (elongation), convexity, and compactness of each soil grain or cluster, using binary image analysis techniques as in [14]. As far as texture is concerned, the fractal dimension of the surface of each soil grain and its local frequency vectors are estimated so as to be used in some further texture analysis and soilsection classification.

The results of granulometric image analysis are also used to study the multiscale structure of soilsections based on their images. The large number of components of such images requires a multisided statistical description of the size distribution of regions. Thus, we use the generalized size histograms to measure many useful attributes including: (1) the average size of grains and pores, expressed by the mean values of the closing- and opening-based, respectively, size histogram; (2) size variability, measured by the deviation around the mean of the size histograms; (3) the percent of grains/pores in localized scale zones; (4) the coarse-tofine ratio; (5) the statistical complexity of grain-pore size distribution, measured by the entropy of the size closingopening histogram; (6) all the above with various alternative 
TABLE 1: The image features (input of the neurofuzzy network).

\begin{tabular}{llll}
\hline & & INPUTS $(X)$ & \\
\hline$x_{1}$ & Mean area (post)* & $x_{8}$ & Mean closing histogram (pre) ${ }^{* *}$ \\
$x_{2}$ & Mean perimeter (post) & $x_{9}$ & Mean opening histogram (pre) \\
$x_{3}$ & Mean eccentricity (post) & $x_{10}$ & St. dev. closing histogram (pre) \\
$x_{4}$ & Mean orientation (post) & $x_{11}$ & St. dev. opening histogram (pre) \\
$x_{5}$ & Mean convexity (post) & $x_{12}$ & Entropy closing histogram (pre) \\
$x_{6}$ & Mean equiv. diam. (post) & $x_{13}$ & Entropy opening histogram (pre) \\
$x_{7}$ & Mean compactness (post) & $x_{14}$ & Void percentage (post) \\
\hline
\end{tabular}

*postsegmentation, ${ }^{* *}$ presegmentation.

interpretations of "scale" based on different geometrical properties (e.g., smallest or largest diameter, area, and degree of connectivity). All the above features extracted from size histograms refer both to the global image as well as to an averaging of its segmented region properties, because the size histogram of the whole image is the sum of the size histograms of individual regions.

As inputs to the neurofuzzy system that will perform the soil classification, we have used, during the first phase of our experiments, only a subset of all the above derived image features shown in Table 1.

\section{CLASSIFICATION AND AUTOMATED CORRESPONDENCE}

Finally, the segmentation results (homogeneous areas), the postsegmentation features (shape and texture) and the granulometric analysis results (size histograms), as well as the biochemical analysis results, are used as inputs in a neurofuzzy system with the objective of classifying the soil into bioecological quality categories. A main difficulty we had was the small number of training data (only 26 input-output pairs), since the chemical analysis of soilsections was expensive and time consuming. Moreover, the dimensionality of the problem was very high (14 features). Thus, the amount of data was not sufficient for "learning from scratch" a neural network to approximate the feature-to-category association. Thus, a two-layered neurofuzzy system is developed, for the hybrid subsymbolic-symbolic processing of the featureto-category association. This neurofuzzy system has the ability to initialize the set of weights with the aid of symbolic information (represented in the form of rules) and then adapt it with the aid of input-output numerical data.

Error minimization on this small number of data will lead to a loss of the generalization property. The symbolic information provided by the experts (bioecologists) must be used in order to improve the system performance. The association of the image features with the quality is essential for the initialization of the neurofuzzy network. Heterogeneity in the soil characteristics implies high biological activity. The features can be associated, either directly or in combination with other features, with the soil fertility of the biological images. The postsegmentation features have a clear physical meaning providing size and textual information and are mainly used for the detection of soil heterogeneous characteristics.

There are many ways to express heterogeneity using the proposed image features (Table 1). Two or more features can form a rule to express the soil key attributes. The disparity of the component size is a significant attribute of a biological image. Mean area $\left(x_{1}\right)$, mean perimeter $\left(x_{2}\right)$, mean equivalent diameter $\left(x_{6}\right)$, and standard deviation of the opening histogram $\left(x_{11}\right)$ are the main features related to the component size. Another attribute is the amount of void in a soil section. The void percentage implies the existence of small components. Consequently, the void percentage $\left(x_{14}\right)$ and the mean area $\left(x_{1}\right)$ can be employed to express the void attribute. In addition, mean convexity $\left(x_{5}\right)$ and mean compactness $\left(x_{7}\right)$ are related to the level of void in a soilsection. Mean orientation $\left(x_{4}\right)$ is slightly relevant to the heterogeneity.

Homogeneous soil characteristics imply low biological activity. The postsegmentation features are mainly involved in detecting high and medium quality biological images. On the other hand, the presegmentation features are very helpful for the detection of low quality images. Entropy closing histogram $\left(x_{12}\right)$, standard deviation of the closing histogram $\left(x_{10}\right)$, and mean closing histogram $\left(x_{8}\right)$ are related to low fertility images. In addition, the existence of uniform large size components refer to homogeneous soil textual characteristics. Mean closing histogram $\left(x_{8}\right)$, mean area $\left(x_{1}\right)$, and entropy closing histogram $\left(x_{12}\right)$ are used for the detection of large components.

The rules relating the features to the bioecological soil quality categories are generally of the form "IF feature (1) and ... and feature (n) THEN category (i)." Each rule consists of an antecedent (its IF part) and a consequence (its THEN part), it is given in symbolic form by the experts and used in order to initialize the neurofuzzy network (giving its initial structure and weights). During the learning process, the weights of both layers may change with the objective of the error minimization approximating the solution of the fuzzy relational equation that describes the association of the input with the output data. After the weight adaptation, the network keeps its transparent structure and the new knowledge represented in it can be extracted in the form of fuzzy IF-THEN rules.

Let $F=\left\{f_{1}, f_{2}, \ldots, f_{n}\right\}$ and $C=\left\{c_{1}, c_{2}, \ldots, c_{m}\right\}$ be the set of features and categories, respectively, and let also 
$R=\left\{r_{1}, r_{2}, \ldots, r_{p}\right\}$ be the set of rules describing the knowledge of the system. The set of antecedents of the rules is denoted by $Z=\left\{z_{1}, z_{2}, \ldots, z_{l}\right\}$. Suppose now that a set $D=$ $\left\{\left(A_{i}, B_{i}\right), i \in \mathbb{N}_{q}\right\}$, where $A_{i} \in \widetilde{F}$ and $B_{i} \in \widetilde{C}$ ( $\widetilde{*}$ is the set of fuzzy sets defined on $*)$, of input-output numerical data is given sequentially and randomly to the system (some of them are allowed to reiterate before the first appearance of some others). The two problems that arise are (1) the initialization of the weights with the aid of fuzzy IF-THEN rules and (2) the adaptation of these weights with the aid of input-output numerical data.

The proposed neurofuzzy system consists of two layers of compositional neurons which are extensions of the conventional neurons [15]. The compositional neurons are based on the operation of triangular norm $T$ [6] and the respective implication operator $\omega_{T}$ defined by

$$
\omega_{T}(a, b)=\sup \{x \in[0,1]: T(a, x) \leq b\}, \quad a, b \in[0,1] .
$$

Based on the above operators, we define the inf- $\omega_{T}$ compositional neuron as

$$
z_{i}=\bigwedge_{j \in \mathbb{N}_{n}} \omega_{T}\left(W^{1}, f_{j}\right), \quad i \in \mathbb{N}_{l}
$$

and the sup- $T$ compositional neuron as

$$
c_{i}=\bigvee_{j \in \mathbb{N}_{l}} T\left(z_{j}, W_{j}^{2}\right), \quad i \in \mathbb{N}_{m}
$$

where $\mathbf{W}^{1}, \mathbf{W}^{2}$ are weight matrices.

The proposed neurofuzzy system uses two layers of compositional neurons. The first consists of inf- $\omega_{T}$ neurons taking as input the features and computing the antecedents of the rules, while the second layer consists of sup- $T$ neurons giving to the output the recognized category. We initialize the weight matrices $\mathbf{W}_{i j}^{1}, i \in \mathbb{N}_{n}, j \in \mathbb{N}_{l}$ and $\mathbf{W}_{i j}^{2}, i \in \mathbb{N}_{n}$, $j \in \mathbb{N}_{l}$, using the set of rules $R$ and taking advantage of the representational power of fuzzy relational equations [15].

The adaptation of the system is based on the computation of the new weight matrices $\mathbf{W}_{\text {new }}^{1}$ and $\mathbf{W}_{\text {new }}^{2}$ for which the error

$$
\epsilon=\sum_{i \in \mathbb{N}_{q}}\left\|B_{i}-c^{i}\right\|
$$

is minimized ( $c^{i}, i \in \mathbb{N}_{q}$ is the network output with input $\left.A_{i}\right)$. The computation is based on the resolution of the fuzzy relational equations

$$
\mathbf{W}^{1} \circ^{\omega_{T}} \mathbf{A}=\mathbf{Z}, \quad \mathbf{Z} \circ^{T} \mathbf{W}^{1}=\mathbf{B},
$$

where $T$ is a continuous $\mathrm{T}$-norm and $\mathbf{Z}$ is the set of antecedents fired when the input $\mathbf{A}$ is given to the network. Using a traditional minimization algorithm (like the steepest descent), we cannot take advantage of the specific character of the problem (symbolic representation). The algorithm that we use is based on a more sophisticated credit assignment that penalizes the neurons of the network using the
TABLE 2: The rules of the neurofuzzy system.

\begin{tabular}{lcl}
\hline $\mathrm{R}$ & Antecedent & \multicolumn{1}{c}{ Output } \\
\hline$r_{1}$ & $x_{1}+x_{2}+x_{6}+x_{10}$ & High fertility \\
$r_{2}$ & $x_{1}+x_{14}$ & High fertility \\
$r_{3}$ & $x_{5}+x_{7}+x_{14}$ & High fertility \\
$r_{4}$ & $x_{3}+x_{4}+x_{10}$ & High fertility \\
$r_{5}$ & $x_{3}+x_{5}$ & Medium fertility \\
$r_{6}$ & $x_{4}+x_{7}+x_{10}$ & Medium fertility \\
$r_{7}$ & $x_{8}+x_{10}+x_{12}$ & Low fertility \\
$r_{8}$ & $x_{1}+x_{9}+x_{13}$ & Low fertility \\
\hline
\end{tabular}

knowledge about the topographic structure of the solution of the fuzzy relation equation [16].

Roughly speaking, the above equations describe a generalized two-layered fuzzy associative memory with the properties of perfect recall and generalization. It has been applied for classifying the six categories of soilsection images into three fertility categories (low, medium, and high fertility). The Juniperus oxycedrus and the Quercus coccifera are classified as high-fertility soil, the Void is classified as low-fertility soil and the rest are classified as medium-fertility soil. For the experiments, we have employed 26 different soilsection images (7 high, 15 medium, and 4 low fertility).

The network has 14 inputs, $X=\left(x_{1}, x_{2}, \ldots, x_{14}\right)$, which were the extracted image features listed in Table 1. It represents eight rules, $R=\left(r_{1}, r_{2}, \ldots, r_{8}\right)$ (see Table 2 ) covering the knowledge provided by the experts. The antecedent and the consequence part are used for the initialization of $\mathbf{W}^{1}$ and $\mathbf{W}^{2}$, respectively.

We first used the Yager T-norm

$$
\begin{aligned}
& Y_{\text {yager }}\left(z, w^{2}\right) \\
& \quad=1-\min \left(1,\left[(1-z)^{p}+\left(1-w^{2}\right)^{p}\right]^{1 / p}\right), \quad p>0,
\end{aligned}
$$

with parameter value $p=2$. The Yager implication $\omega_{T}$ is

$$
Z_{\text {yager }}\left(w^{1}, x\right)= \begin{cases}1-\left[\left(1-w^{1}\right)^{p}-(1-x)^{p}\right]^{1 / p}, & w^{1}>x \\ 1, & \text { else. }\end{cases}
$$

The neurons were adapted independently, in 20 iterations. The adaptation procedure did not alter the knowledge of the system, it only adjusted the strength of the image features. The error performance is illustrated in Figure 5. Although the number of numerical data was not sufficient to learn the neural network from scratch, the adaptation of the system has been performed using the data set presented in the previous section (we excluded one data from each category and used it for testing). Before the adaptation procedure the classification rate was $70 \%$, while afterwards it rose to $80 \%$. In general, we could achieve a better performance by importing more rules in the network. However, the number of rules influenced the generalization and symbolic meaning of the network. 


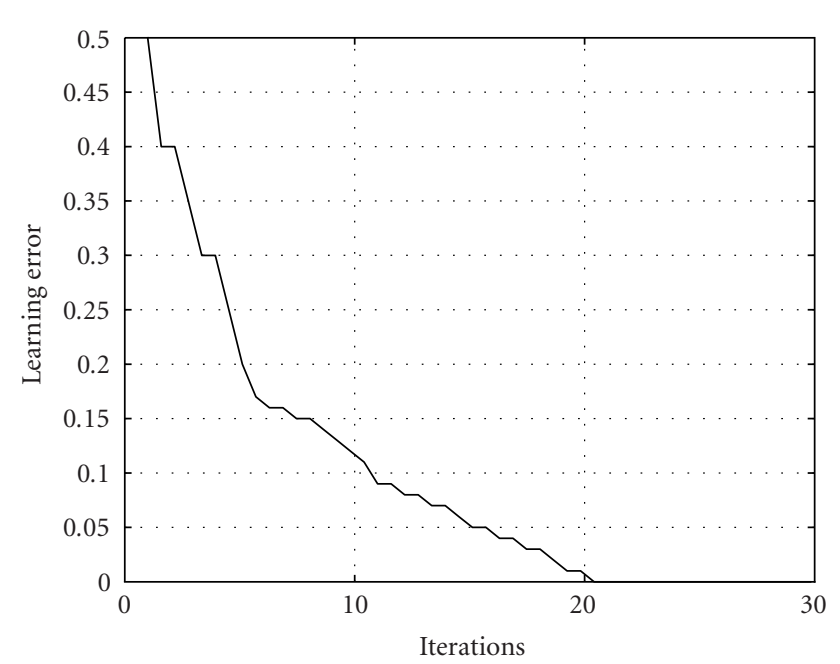

FIgURE 5: The error performance of the system.

\section{CONCLUSION}

In this paper, we have developed the first phase of an automated system for soil image analysis and quality inference. The image analysis was based on relatively advanced techniques that emphasized object-oriented processing, but the final features used for classification were of a simple type to maintain a modest overall complexity of the system. In future phases, we plan to use more sophisticated visual features resulting from geometrical and statistical object-based shape and texture analysis as well as integrate into the neuro-fuzzy inference procedure a more mature reasoning and a finer grading for the soil quality from the bioecology experts.

\section{ACKNOWLEDGMENTS}

We wish to thank the additional researchers who participated in this research project. (1) D. Dimitriadis, A. Doulamis, N. Doulamis, G. Tsechpenakis from NTUA. (2) J. Diamantopoulos, M. Argyropoulou from Dept. Biology, Arist. Univ. Thessaloniki. (3) S. Varoufakis, N. Vassilas, C. Tzafestas from NCSR Demokritos, Athens. This research work was supported by the Greek General Secretariat for Research and Technology and by the European Union under the program חENE $\Delta$-2001 with Grant \# 01E $\Delta 431$. It was also partially supported by the European Network of Excellence "MUSCLE."

\section{REFERENCES}

[1] P. Bullock, N. Fedoroff, A. Jongerius, G. Stoops, and T. Tursina, Eds., Handbook for Soil Thin Section Description, Waine Research Publications, Albrighton, Wolverhampton, UK, 1985.

[2] H. J. A. M. Heijmans, Morphological Image Operators, Academic Press, New York, NY, USA, 1994.

[3] P. Salembier and J. Serra, "Flat zones filtering, connected operators, and filters by reconstruction," IEEE Trans. Image Processing, vol. 4, no. 8, pp. 1153-1160, 1995.

[4] L. Vincent, "Morphological grayscale reconstruction in im- age analysis: applications and efficient algorithms," IEEE Trans. Image Processing, vol. 2, no. 2, pp. 176-201, 1993.

[5] F. Meyer and P. Maragos, "Nonlinear scale-space representation with morphological levelings," Journal of Visual Communication and Image Representation, vol. 11, no. 2, pp. 245-265, 2000.

[6] G. J. Klir and B. Yuan, Fuzzy Sets and Fuzzy Logic: Theory and Applications, Prentice-Hall, Upper Saddle River, NJ, USA, 1995.

[7] P. Maragos, V. Tzouvaras, and G. Stamou, "Synthesis and applications of lattice image operators based on fuzzy norms," in Proc. IEEE International Conference on Image Processing, vol. 1, pp. 521-524, Thessaloniki, Greece, October 2001.

[8] J. Serra, Image Analysis and Mathematical Morphology, Academic Press, New York, NY, USA, 1982.

[9] P. Maragos, "Pattern spectrum and multiscale shape representation," IEEE Trans. on Pattern Analysis and Machine Intelligence, vol. 11, no. 7, pp. 701-716, 1989.

[10] A. Doulamis, N. Doulamis, and P. Maragos, "Generalized multiscale connected operators with applications to granulometric image analysis," in Proc. IEEE International Conference on Image Processing, vol. 3, pp. 684-687, Thessaloniki, Greece, October 2001.

[11] P. Maragos and A. C. Bovik, "Image demodulation using multidimensional energy separation," Journal of the Optical Society of America $\{A\}$, vol. 12, no. 9, pp. 1867-1876, 1995.

[12] S. Beucher and F. Meyer, "The morphological approach to segmentation: the watershed transformation," in Mathematical Morphology in Image Processing, E. R. Dougherty, Ed., pp. 433-481, Marcel Dekker, New York, NY, USA, 1993.

[13] A. Sofou, C. Tzafestas, and P. Maragos, "Segmentation of soilsection images using connected operators," in Proc. IEEE International Conference on Image Processing, vol. 3, pp. 10871090, Thessaloniki, Greece, October 2001.

[14] R. M. Haralick and L. G. Shapiro, Computer and Robot Vision, Addison-Wesley, Reading, Mass, USA, 1992.

[15] G. B. Stamou and S. G. Tzafestas, "Fuzzy relation equations and fuzzy inference systems: an inside approach," IEEE Trans. Systems, Man, and Cybernetics, vol. 29, no. 6, pp. 694702, 1999.

[16] G. B. Stamou and S. G. Tzafestas, "Neural fuzzy relational systems with a new learning algorithm," Mathematics and Computers in Simulation, vol. 51, no. 3, pp. 301-314, 2000.

Petros Maragos received his Ph.D. from Georgia Tech in 1985. During 1985-1998, he worked as a Professor of electrical and computer engineering at Harvard University and Georgia Tech in the USA. Since 1998, he has been working as a Professor at the National Technical University of Athens (NTUA). His research interests include image processing and computer vision and speech processing and recognition.

Anastasia Sofou received her first degree in 1998 from the Department of Informatics, University of Athens, Greece, and her M.S. in advanced computing in 1999 from University of Bristol, United Kingdom. She is currently pursuing her Ph.D. in the area of computer vision at the National Technical University of Athens. Her research interests include computer vision, image processing, image segmentation, and pattern recognition.
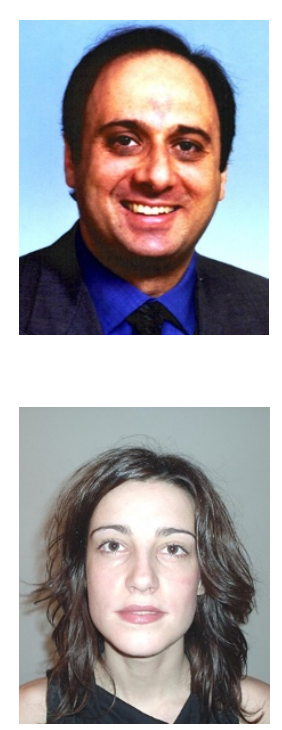
Giorgos B. Stamou obtained the Diploma and Ph.D. degrees in electrical and computer Engineering from the National Technical University of Athens (NTUA) in 1994 and 1998, respectively. He is currently a Senior Researcher at the NTUA Institute for Communication and Computer Systems. His research interests include fuzzy set theory and decision making, neural networks, hybrid intelligent systems, computer vision,

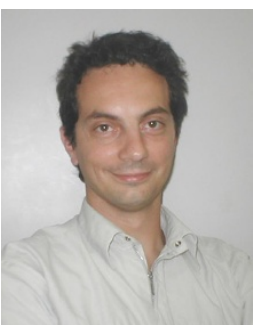
semantic video analysis, and human computer interaction.

Vassilis Tzouvaras received his B.S. degree in 1998 from University of Essex, United Kingdom and his M.S. degree in 1999 from University of Sheffield, United Kingdom. $\mathrm{He}$ is currently pursuing his Ph.D. at the $\mathrm{Na}-$ tional Technical University of Athens. His research interests are neural networks, computer vision, fuzzy systems and inferencing, and semantic video analysis.

Efimia Papatheodorou is a Biologist with a background in soil ecology. She works as a Lecturer in the Department of Ecology, in the Biology Department of Thessaloniki University. Her work relates to the evaluation of soil quality, in terms of biological and biochemical parameters, in physical and agricultural ecosystems.

George P. Stamou has a great experience in soil biology. His work relates to population dynamics and community organization patterns of soil animals, effects of global climate change on soil environments, desertification of Mediterraneantype ecosystems, and effect of management practices and land use on soil communities.
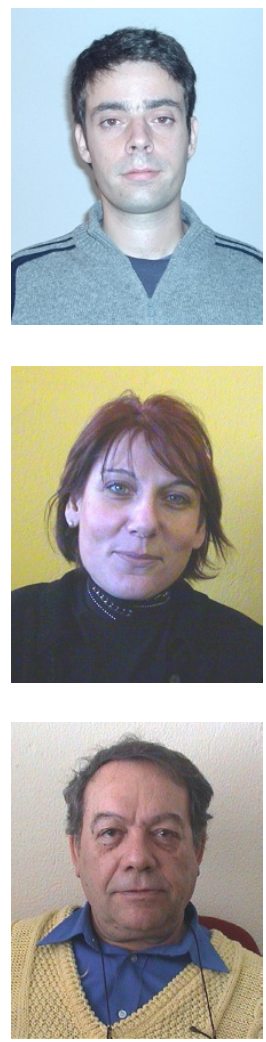